\title{
Severe virilization in adolescent girl with Sertoli-Leydig cell tumor - case report and review of the literature
}

Nevena Krnic ${ }^{1}$, Natasa Rojnic Putarek ${ }^{1}$, Katja Dumic Kubat ${ }^{1}$, Mihajlo Strelec ${ }^{2}$, Anita Spehar Uroic ${ }^{1}$, Marija Macan ${ }^{3}$, Lana Skrgatic ${ }^{2}$, Vesna Kusec ${ }^{4}$

${ }^{1}$ Division of Pediatric Endocrinology and Diabetes, Department of Pediatrics, University Hospital Center Zagreb, University of Zagreb Medical School, Zagreb, Croatia, ${ }^{2}$ Human Reproduction Unit, Department of Gynecology and Obstetrics, University Hospital Center, Zagreb, Croatia, University of Zagreb Medical School, Zagreb, Croatia, ${ }^{3}$ Department of Pathology and Cytology, University Hospital Center Zagreb, Zagreb, Croatia, ${ }^{4}$ Endocrinological Laboratory, Department of Laboratory Medicine, University Hospital Centre Zagreb, University of Zagreb Medical School, Zagreb, Croatia

Correspondence: nevena@krnich.com

Tel.: + 38512388532

Fax.:+ 38512421535

Received: November 29, 2017

Accepted: January 5, 2018

Key words: Sertoli-Leydig cell tumor - Sex cord-stromal tumor - Virilization - Alpha fetoprotein.

\section{Introduction}

Sertoli-Leydig cell tumor (SLCT) belongs to a group of sex cord-stromal tumors and is a rare neoplasm, representing less than $0.2 \%$ of ovarian tumors (1). Although most common in women in the $3^{\text {rd }}$ decade of life (1),
Objective - The aim was to report a case of a 17-year-old girl with marked virilization and secondary amenorrhea due to androgen producing Sertoli-Leydig cell tumor (SLCT). We also provided a brief review of the literature with insight into clinical picture, pathological features and treatment of patients with this rare tumor. Case report - We present a 17-year-old girl with SLCT, who developed clinical symptoms of severe virilization. The tumor secreted testosterone and alpha fetoprotein (AFP). The imaging revealed tumor of the right ovary which histologically corresponded to intermediate-grade SLCT with low mitotic rate. Furthermore, it was stage IA according to International Federation of Gynecology and Obstetrics. Laparoscopic right salpingo-oophorectomy was preformed, resulting in regression of virilization symptoms and normalization of serum testosterone and AFP levels. Conclusion - Due to rarity of SLCT in children and adolescents, gathering information from available case reports is necessary to establish guidelines regarding optimal management, including type of surgery and adjuvant chemotherapy. New knowledge regarding related diseases in SLCT survivors emphasize the need for long-term follow up of these patients.

it can be seen in all age groups (2). In 50$70 \%$ of cases, SLCT presents as an abdominal mass, and $30-50 \%$ of them are hormonally active, producing mostly androgens and rarely estrogen $(1,3)$. Occasionally, SLCT produce alpha fetoprotein (AFP) $(1,2)$. Elevated serum testosterone levels may lead to virilization, including hirsutism, acne, voice hoarseness, oligo- or amenorrhea, hypertrophy of the clitoris, and breast atrophy (3). These tumors are usually unilateral. Histologically, they consist of variable proportions of Sertoli and Leydig cells and are classified as 
well, intermediate or poorly differentiated tumors. Intermediate and poorly differentiated Sertoli-Leydig cell tumors can show retiform pattern and contain heterologous elements which include epithelial and/or mesenchymal tissues, as well as tumors arising from these elements (4). The treatment consists of surgery followed by adjuvant chemotherapy in metastatic or poorly differentiated tumors. The overall prognosis is good, with five-year survival rate of $70-90 \%$. Disseminated or poorly differentiated tumors are associated with a worse prognosis $(2,5)$. Due to low incidence of SLCT, optimal management is not well established.

The aim was to report a case of a 17-yearold girl with marked virilization and secondary amenorrhea due to androgen producing SLCT. Furthermore, a brief review of literature was provided with insight into clinical picture, pathological features and treatment of patients with this rare neoplasm.

\section{Case report}

The patient is a 17-years old nulliparous female with secondary amenorrhea for 1.5 years, as well as progressive voice hoarsening, acne and hirsutism. She had no history of abdominal bloating or pain. Initial laboratory evaluation detected elevated basal levels of 17-hydroxyprogesterone $(12.8 \mathrm{nmol} / \mathrm{L}$; reference range, 0.6-2.3). Nonclassical congenital adrenal hyperplasia (CAH) was suspected and patient was given hydriocortisone at an outside insitution. During hydrocortisone therapy she remained amenorrhoic and her symptoms persisted. Upon initial assessment the enlarged thyroid was noted, but further evaluation, including thyroid hormones, antibodies, ultrasound, fine needle aspiration cytology, and scintigraphy, was all normal.

Initial ovarian ultrasound revealed cystic mass in the projection of the right ovary measuring $65 \times 40 \mathrm{~mm}$. Uterus and the left ovary were unremarkable. Abdominal MRI was performed revealing partially solid and partially cystic right adnexal mass measuring $53 \times 48 \times 50 \mathrm{~mm}$. The patient was then referred to our institution for further workup. Upon admission, physical examination revealed a girl with muscular appearance, severe hirsutism (Ferriman-Gallwey score 22), with acne on face and the upper body, hypotrophic breasts, clitoromegaly, and hoarse voice. The treatment with hydrocortisone was discontinued. Laboratory evaluation revealed significantly elevated serum testosterone and AFP levels, while other tumor markers were within the normal range. The laboratory results are shown in Table 1 .

Transrectal ultrasound showed small uterus displaced to the left, normal morphology of the left ovary and globular tumor of the right ovary, measuring $60 \mathrm{~mm}$ in diametar, which was predominantly solid, and showed no changes in Color Doppler blood flow. The patient underwent laparoscopic right salpingo-oophorectomy. The right ovary was markedly enlarged and incorporated in tumorous mass measuring $85 \times 70 \times 20 \mathrm{~mm}$, with smooth white surface. On inspection, the other abdominal structures appeared unremarkable and peritoneal diagnostic lavage was done for cytology. Macroscopically, the ovarian tumor had solid, white-yellow to brown cut surface. Histopathological examination showed that the tumor was composed of Sertoli and Leydig cells forming solid areas, cords and tubules, some of which were cystically dilated. Immunohistochemical staining was diffusely positive for inhibin and calretinin, focally positive for cytokeratin CKAE1/AE3, and positive for Melan A in Leydig cells. Moreover, alfa-fetoprotein and hepatocyte antigen immunohistochemistry showed scattered positivity in some tumor cells. The tumor was classified as Sertoli-Leydig cell tumor with intermediate differentiation, and was staged as IA according to International Federation 


\begin{tabular}{|c|c|c|c|c|}
\hline Laboratory findings & At diagnosis & 12 days following surgery & 7 months following surgery & Reference range \\
\hline Testosterone (nmol/L) & 7.6 & 2.0 & 0.21 & $0.4-1.9$ \\
\hline Free testosterone $(\mathrm{pmol} / \mathrm{L})$ & 232 & 56.6 & 5.45 & $3.5-30$ \\
\hline Alpha-fetoprotein $(\mathrm{mcg} / \mathrm{L})$ & 85.7 & 15.4 & 2.77 & $<7.0$ \\
\hline $\begin{array}{l}\text { Human chorionic } \\
\text { gonadotropin (IU/L) }\end{array}$ & Negative & - & Negative & Negative \\
\hline Cancer antigen 125 (kIU/L) & 12.9 & - & 13.19 & $<35.0$ \\
\hline Cancer antigen 19-9 (kIU/L) & 9.6 & - & - & $<37.0$ \\
\hline
\end{tabular}

of Gynecology and Obstetrics (FIGO). The mitotic rate was low (2 mitosis per 10 high power fields). No necrosis or vascular invasion was seen. Cytological analysis of peritoneal fluid showed no evidence of tumor.

The possibility of adjuvant chemotherapy was discussed with the patient and her family, but they opted not to administer it. The levels of testosterone and AFP were slightly above the normal range 12 days after surgery, but normalized during the follow up. Menstrual cycles were restored one month following surgery, but have remained irregular. Six months after surgery hirsutism and acne regressed, the voice was less harsh and the size of clitoris was reduced. The patient remains in remission one year after surgery with normal laboratory findings and ovarian ultrasound.

\section{Discussion}

Herein, we present an adolescent girl with a rare sex cord-stromal tumor, SLCT with clinical symptoms of severe virilization and elevated AFP levels. The tumor was successfully treated by laparoscopic surgery. Although the secretion of AFP in SLCT was considered rare, recent reports show significant proportion of SLCT with elevated AFP levels, particularly in children and adolescents, ranging from 14 to $27 \%(1,2,6)$. The secretion of AFP makes it difficult to distinguish SLCT from other tumors that usually produce AFP
(7). Therefore, when evaluating patients with elevated levels of AFP, SLCT should always be considered, particularly if signs of virilization are present.

Immunohistochemical staining can be positive for AFP in Leydig or Sertoli cells, intestinal-type mucinous epithelium, hepatocytes or hepatocyte-like cells. In our patient, the AFP staining was positive in scattered heterologous hepatocyte-like cells. Similar to previous reports, moderately elevated AFP levels in our patient normalized soon after surgery. However, AFP could serve as a marker of tumor recurrence $(7,8)$. Due to relatively rare occurrence of SLCT in children and adolescents, optimal management and follow up were not well established in these patients, particularly in cases with unfavorable histology. In children and young females, fertility sparing surgery is the treatment of choice. In patients with FIGO stage I and favorable histology, unilateral salpingo-oophorectomy is curative. Metastases to lymph nodes are rare, so lymphadenectomy is not considered a routine part of the current operative procedure (9). In majority of reported cases laparotomy was performed, however, minimally invasive laparoscopic surgery has been suggested as preferred modality by several authors $(1,3)$, and this approach was used in our patient. Laparoscopy reduces morbidity and recovery time, but might increase the risk of intraoperative tumor rupture according to some authors $(3,6)$. The European Cooperative 
Study Group on Pediatric Rare Tumors (EXPeRT) demonstrated high tendency of SLCT relapse in cases of intraoperative dissemination (2). Therefore, precautions should be made to prevent tumor spillage during surgery. The enucleation of the tumor in toto by using endoscopic bag assures protection against tumor spillage (6).

Adjuvant chemotherapy is recommended for patients with advanced disease, intermediate and poorly differentiated tumors, retiform pattern, presence of heterologous elements, and in cases of pre- or intraoperative dissemination (FIGO stage Ic) $(1,2)$. Due to the presence of heterologous elements on histology, adjuvant chemotherapy was proposed to our patient, however, she refused it. In largest cohort of children with SCLT reported by EXPeRT Study Group, the most common presenting symptoms were virilization, hirsutism (10/41) and secondary amenorrhea (8/41), while $17 / 41$ patients reported abdominal pain (2). Our patient did not experience any abdominal symptoms, probably due to relatively small size of the tumor, while clinical symptoms of virilization were considerable. In study conducted by Fresneau et al., that included 11 patients with SCLT, the presence of endocrine symptoms correlated with younger age at diagnosis and smaller tumor size (10). It is difficult to predict the long-term clinical nature of the disease, particularly in terms of different histology. The prognosis depends on tumor staging, differentiation, presence of heterologous elements, mitotic index, and tumor dissemination before, or during surgery (11). Most of the children with SLCT present with intermediate-grade tumor (2). While Roth et al. (12) found younger age to be associated with less differentiated tumors, Sigismondi et al. (1) found no correlation between patients' age and occurrence of well, intermediate and poorly differentiated tumor.
In EXPeRT study, none of the patients with FIGO stage IA (24/44) developed tumor relapse regardless of the type of differentiation. However, in FIGO stage IC and higher, the poor outcome was correlated with lower grade of histologic differentiation (2). Patients with well-differentiated tumor had a 5-year survival rate of $100 \%$, while those with intermediate and poorly differentiated tumors $77.8 \%$. Five-year overall survival rate was $92.3 \%$ for FIGO stage I and $33.3 \%$ for higher FIGO stages (1). The recurrence of malignant SLCTs usually occurs 1 to 3 years following the initial diagnosis. The tumor recurrence after five years of follow-up is rare $(1,2,10)$. The reports on the follow up of patients with SLCT are promising regarding fertility potential, as there are emerging reports of successful pregnancies following SLCT treatment (13). Recent data emphasize the risk of development of thyroid disease in SLCT survivors, particularly thyroid carcinoma. This is attributed to association of SLCT with DICER1 mutations $(14,15)$. Kato et al. (14) demonstrated that SCLT patients, carriers of DICER1 mutations are younger, with marked virilization and heterologous components in tumor, compared to DICER1 wildtype SLCT patients. Two patients followed by EXPeRT study group developed thyroid carcinoma. They were not tested for DICERI mutation (2).

Prior to the diagnosis of SLCT, our patient was evaluated for thyroid disease because of palpable neck mass. Since she has not been tested for DICER1 mutations, her follow up should include routine thyroid palpation, thyroid hormone measurement and yearly thyroid ultrasound evaluation. Moreover, it is advisable to perform DICER1 mutation analysis, since carriers of these mutations can develop contralateral, metachronous ovarian tumors even after the usual period of recurrence (15). Furthermore, their offspring carry increased risk for development of pleuropul- 
monary blastoma (15). Our patient requires prolonged follow up due to emerging knowledge regarding predisposition of patients with SLCT to develop other malignancies. The multidisciplinary approach is needed to establish the correct diagnosis and to determine optimal management of each patient. Long-term follow-up is necessary due to evolving insights regarding related diseases in SLCT survivors.

\section{Conclusion}

Sertoli-Leydig cell tumors are rare in children and adolescents and current recommendations regarding surgical treatment and chemotherapy rely on published single case reports and case control studies. Special note should be put on long-term follow up of SLCT survivors and their family members since recent literature suggests that SLCT could be a part of hereditary tumor susceptibility syndrome with a variety of manifestations.

Authors' contributions: Conception and design: NK, NRP and VK; Acquisition, analysis and interpretation of data: KDK, ASU, LS, MS and MM; Drafting the article: NK, VK, NRP, LS, KDK and ASU; Revising the article critically for intellectual content: NK, NRP, and VK; Approved final version of the manuscript: NK, NRP, KDK, ASU, LS, MS, MM and VK.

Conflict of interest: The authors declare that they have no conflict of interest.

\section{References}

1. Sigismondi C, Gadducci A, Lorusso D, Candiani M, Breda E, Raspagliesi F, et al. Ovarian SertoliLeydig cell tumors. A retrospective MITO study. Gynecol Oncol. 2012;125(3):673-6.

2. Schneider DT, Orbach D, Cecchetto G, Stachowicz-Stencel T, Brummel B, Brecht IB, et al. Ovarian Sertoli Leydig cell tumours in children and adolescents: an analysis of the European Cooperative Study Group on Pediatric Rare Tumors (EXPeRT). Eur J Cancer. 2015;51(4):543-50.
3. Litta P, Saccardi C, Conte L, Codroma A, Angioni S, Mioni R. Sertoli-Leydig cell tumors: current status of surgical management: literature review and proposal of treatment. Gynecol Endocrinol. 2013;29(5):412-7.

4. Outwater EK, Wagner BJ, Mannion C, McLarney JK, Kim B. Sex cord-stromal and steroid cell tumors of the ovary. Radiographics. 1998;18(6):1523-46.

5. Schultz KA, Schneider DT, Pashankar F, Ross J, Frazier L. Management of ovarian and testicular sex cord-stromal tumors in children and adolescents. J Pediatr Hematol Oncol. 2012;34:S55-63.

6. Fauvet R, Boccara J, Dufournet C, Poncelet C, Daraï E. Laparoscopic management of borderline ovarian tumors: results of a French multicenter study. Ann Oncol. 2005;16:403-10.

7. Al-Hussaini M, Al-Othman Y, Hijazi E, McCluggage WG. A Report of Ovarian Sertoli-Leydig Cell Tumors With Heterologous Intestinal-type Glands and Alpha Fetoprotein Elevation and Review of the Literature. Int J Gynecol Pathol. 2017 Jun 2. doi: 10.1097/PGP.0000000000000408.

8. Poli UR, Swarnalata G, Maturi R, Rao ST. Recurrent alphafetoprotein secreting Sertoli-Leydig cell tumor of ovary with an unusual presentation. Indian J Cancer. 2009;46(1):64-6.

9. Brown J, Sood AK, Deavers MT, Milojevic L, Gershenson DM. Patterns of metastasis in sex cordstromal tumors of the ovary: can routine staging lymphadenectomy be omitted? Gynecol Oncol. 2009;113(1):86-90.

10. Fresneau B, Orbach D, Faure-Conter C, Verité C, Castex MP, Kalfa N, Martelli H, Patte C. Sex-Cord Stromal Tumors in Children and Teenagers: Results of the TGM-95 Study. Pediatr Blood Cancer. 2015;62:2114-9.

11. Demidov VN, Lipatenkova J, Vikhareva O, Van Holsbeke C, Timmerman D, Valentin L. Imaging of gynecological disease: clinical and ultrasound characteristics of sertoli cell tumors, sertoli-leydig cell tumors and leydig cell tumors. Ultrasound $\mathrm{Ob}-$ stet Gynecol. 2008;31(1):85-91.

12. Roth LM, Anderson MC, Govan AD, Langley FA, Gowing NF, Woodcock AS. Sertoli-Leydig cell tumors: a clinicopathologic study of 34 cases. Cancer. 1981;48(1):187-97.

13. Stavrakis T, Kalogiannidis I, Petousis S, Tsompanidou C, Delkos D, Prapas N, et al. Fertility-sparing management and obstetric outcomes in a 20 -yearold patient with a Sertoli-Leydig cell tumor of the 
ovary: A case report and review of the literature. Oncol Lett. 2016;12(2):1079-82.

14. Kato N, Kusumi T, Kamataki A, Tsunoda R, Fukase M, Kurose A. DICER1 hotspot mutations in ovarian Sertoli-Leydig cell tumors: a potential association with androgenic effects. Hum Pathol. 2017;59:41-7.
15. Schultz KA, Harris A, Williams GM, Baldinger S, Doros L, Valusek P, et al. Judicious DICER1 testing and surveillance imaging facilitates early diagnosis and cure of pleuropulmonary blastoma. Pediatr Blood Cancer. 2014;61(9):1695-7. 